\title{
Tight Glycemic Control - The leading role of insulin sensitivity in determining efficacy and thus outcome
}

\author{
J. Geoffrey Chase*, Aaron J. Le Compte*, Geoffrey M. Shaw**, Jessica Lin**, Christopher G. Pretty*, Normy Razak*, \\ Jacquelyn Parente*, Adrienne Lynn***, Christopher E. Hann* and Fatanah Suhaimi* \\ *University of Canterbury, Dept of Mechanical Engineering, Christchurch, New Zealand \\ (Tel: +64-3-364-7001; e-mail: geoff.chase@canterbury.ac.nz). \\ **University of Otago, School of Medicine, Christchurch, New Zealand \\ ***Neonatal Unit, Christchurch Women's Hospital, Christchurch, New Zealand
}

\begin{abstract}
Tight glycaemic control (TGC) has emerged as a major focus in critical care. However, repeating the initial successful reductions in reducing mortality and other outcomes via TGC has proven very difficult. Hence, there has been growing debate over the necessity of TGC, its goals, safety from hypoglycemia, and target cohorts. This article reviews existing knowledge and results to provide a new interpretation and explanation for the variable results in applying TGC. It then uses a validated metabolic system model to show how the root cause is the intra- and inter- patient variability, which makes TGC difficult over diverse cohorts and thus yields such variable results over many protocols.
\end{abstract}

Keywords: Critical Care, Glycemic Control, Variability, Modeling, Insulin Sensitivity, Modeling.

\section{INTRODUCTION}

\subsection{The Physiological and Clinical Problem}

Critically ill patients often experience stress-induced hyperglycemia and high insulin resistance (McCowen et al., 2001). It is strongly associated with increased mortality (Krinsley, 2003). Hyperglycaemia is also associated with increases in other negative clinical outcomes, including infection (Bistrian, 2001), sepsis and septic shock (Das, 2003, Marik and Raghavan, 2004), myocardial infarction (Capes et al., 2000), and polyneuropathy and multiple-organ failure (Van den Berghe et al., 2001).

In specific, a strong counter-regulatory (stress) hormone response is further aggravated by pro-inflammatory immune responses (Fernandez-Real et al., 2003), which stimulate endogenous glucose production (EGP) and can inhibit insulin production and/or action to a variable extent, thus increasing insulin resistance. Absolute and relative insulin deficiency is a further cause. Finally, high glucose content nutritional regimes exacerbate hyperglycemia and thus mortality (Krishnan et al., 2003), whereas reducing glucose intake has reduced glycemic levels (Ahrens et al., 2005).

The problem is summarised as a strong counter-regulatory hormone driven stress response that induces significant insulin resistance and can antagonise insulin production and action. Coupled with unsuppressed EGP and potentially excessive nutritional inputs, high blood glucose is inevitable. Dynamic patients whose condition, and thus insulin resistance, evolves regularly and sometimes acutely, provide a further challenge to providing consistent TGC across a every individual patient in a cohort.

\subsection{Hyperglycaemia, TGC and Outcome}

Van den Berghe et al (2001), obtained significant mortality reductions for a cardiovascular surgery cohort, as well as reducing other outcomes and treatments. It was matched by the retrospective study of Krinsley (2004). Van den Berghe et al (2006) was less successful with a more dynamic medical ICU cohort. Finally, the SPRINT study obtained significant mortality reductions for a medical ICU cohort controlling both nutrition and insulin inputs (Chase et al., 2008b). However, several studies got no similar result (Treggiari et al., 2008, De La Rosa et al., 2008, Meijering et al., 2006), with some stopped for safety due to hypoglycemia (Brunkhorst et al., 2008, Devos and Preiser, 2007).

Hence, there is significant controversy around TGC and its application (Wilson et al., 2007, Mesotten, 2008, Finfer and Delaney, 2008, Devos and Preiser, 2007). This paper posits that it is a lack of understanding of both the problem and the patient dynamics that hinder clarity on all of these issues.

\section{KNOWN FACTS AND HOW TGC REALLY WORKS}

The following are well reported in this area:

- Mortality increases with mean, maximum or range of blood glucose, particularly in uncontrolled cohorts (Krinsley, 2003, Umpierrez et al., 2002).

- Mortality increases with blood glucose variability, independent of mean or median value (Egi et al., 2006, Krinsley, 2008).

- Blood glucose levels over 7.0-8.0 mmol/L reduce and/or eliminate the effectiveness immune response to infection (Weekers et al., 2003, Fernandez-Real et al., 2003). 
This article would add the following points to consider:

- Mortality is an individual outcome, and not a cohort outcome. Even if its rate is measured by cohort.

- Patients are individual and dynamic in their condition, with glucose levels or/and insulin resistance being a marker of severity of disease (Chase et al., 2006).

- Thus, glucose response is an individual outcome.

Specifically, the benefits of TGC work at an individual level. Only those patients who are tightly controlled will receive benefit. Hence, TGC is effective for a cohort only if it is (relatively) effective for every patient. In contrast to the same cohort result with a wide spread in TGC efficacy. Thus, it implies that per-patient results, rather than cohort based results, should be the goal of any TGC protocol.

Only a few trials have reported per patient results (Chase et al., 2008b, Goldberg et al., 2004, Van den Berghe et al., 2003). More importantly, Table 1 summarises the cohort results from several trials in terms of median and (lognormal multiplicative) variance. It clearly shows that there is no clear correlation between clinical "success" and glycemia.

Table 1: Cohort-based clinical trial results. Where Leuven 2001/2006, Krinsley and SPRINT were "successful". Control or retrospective groups are all assumed "unsuccessful".

\begin{tabular}{|l||c|c|}
\hline Trial & $\begin{array}{c}\text { Median } \\
(\mathbf{m m o l} / \mathbf{L})\end{array}$ & $\begin{array}{c}\text { Variance } \\
\boldsymbol{\sigma}^{*}\end{array}$ \\
\hline \hline Leuven 2001 & 5.6 & 1.20 \\
Leuven 2001 Control & 8.3 & 1.24 \\
Krinsley & 6.7 & 1.50 \\
Krinsley Retrospective & 7.2 & 1.76 \\
Leuven 2006 - all & 6.0 & 1.29 \\
Leuven 2006 Control - all & 8.3 & 1.22 \\
Leuven 2006 - LoS $\geq 3$ day & 5.8 & 1.27 \\
Leuven 2006 Cont. - LoS $\geq 3$ day & 8.6 & 1.17 \\
Treggiari et al - Control / None & 7.7 & 1.30 \\
Treggiari et al - 4-7 mmo/L goal & 7.5 & 1.28 \\
Treggiari et al - 4-6 mmol/L goal & 7.0 & 1.26 \\
SPRINT & 5.8 & 1.24 \\
SPRINT Retrospective & 7.2 & 1.88 \\
VISEP IIT all & 6.1 & 1.17 \\
VISEP Conventional all & 8.2 & 1.24 \\
\hline
\end{tabular}

* All values converted to lognormal median (geometric mean) and multiplicative variance $\left(\sigma^{*}\right)$

In particular, it should be noted that SPRINT statistically decoupled all glucose metrics (mean, variability, peak/range) from mortality across the TGC cohort $(\mathrm{p}<0.05)$. Thus, there was no relationship between any glucose metric and mortality, meaning that survivors and non-survivors received equivalent tight control over all patients.

More succinctly, TGC with SPRINT eliminated glycemia as an indicator of mortality over a 384 patient cohort. Given that the SPRINT TGC group had lower mortality than the retrospective comparison cohort, it can be directly assumed that no other risk factor increased (significantly enough) to offset this decoupling of glycemia and mortality. In specific, it can thus be assumed that SPRINT reduced mortality directly via TGC.

Finally, the only other study that analysed glucose and mortality within the TGC cohort still showed a link between them (Van den Berghe et al., 2003).

As a statistical note, Table 1 uses lognormal statistics because TGC data is often skewed. While arithmetic mean is typically used to report central tendency, it is not a robust statistic, as it is greatly influenced by outliers. For skewed distributions, the arithmetic mean will not match the notion of "middle", and robust lognormal or non-parametric statistics provide a much better definition of central tendency.

There are three direct conclusions that can be drawn:

1. Conclusion 1: It is per-patient glycemic results that are important, rather than over a cohort, since mortality is an individual response to condition and therapy.

2. Conclusion 2: The true goal of TGC should be to statistically decouple glycemia (mean, variability, etc) from mortality across a tightly controlled cohort.

3. Conclusion 3: Median blood glucose levels should be less than $\sim 7.0 \mathrm{mmol} / \mathrm{L}$, and thus allow for reasonable variation in control as patient condition evolves.

The first point thus asks how does the median patient and their associated $5^{\text {th }}, 25^{\text {th }}, 75^{\text {th }}$ and $95^{\text {th }}$ percentile compatriots respond to TGC? This answer will determine whether all patients in a cohort, regardless of outcome, receive the same level of control, which is critical to making any comparison. In particular, comparing mortality between a TGC and control or retrospective cohort implicitly assumes that the control received within each cohort was equivalent for all patients, thus rendering the comparison of mortality valid. If this assumption doesn't hold then it is not possible to determine the impact of TGC or glycemic control in general, because it is not possible to verify that survivors or nonsurvivors in either cohort had a significantly different glycemic outcome.

The second point implies that the real goal of TGC should be assessed for each patient, rather than as a cohort. Decoupling glycemia from mortality will not likely raise mortality given the evidence, and any such a rise would thus be indicative of other factors outside of the TGC intervention. It has the added advantages of eliminating the given ICUs mortality rate before TGC, as it considers only the TGC cohort, thus also simplifying trial design and cohorts required.

The last point simply follows from studies on immune response efficacy in hyperglycemia. This paper recommends a median target of $7.0 \mathrm{mmol} / \mathrm{L}$ or less to allow for variation and patient evolution, while keeping blood glucose less than $8.0 \mathrm{mmol} / \mathrm{L}$. However, given the association between glycemia and severe infection, lower may be better.

Summary: These conclusions imply that measuring glycemic control over entire cohorts for comparison is not valid. Critically, most prior studies have not done the analyses to determine whether their TGC protocols were effective 
enough across all types of patient to provide a valid comparison to mortality in the randomised control or retrospective cohorts used.

Thus, the goal of any TGC protocol should be to (first) decouple glycemia from outcome in the TGC cohort, before comparison to a control. If mortality is thus lower under TGC, it can be assumed that other indices of risk were not (significantly) elevated enough to offset them.

The remaining question: How to achieve such tight control and/or conversely, what physiological behaviours have made these goals difficult to achieve in practice.

\section{INSULIN SENSITIVITY \& PATIENT-SPECIFIC TGC}

Glycemia in the critically ill generally reflects patient condition. The more critically ill, the more variable and greater their glycemia, as seen in several studies (Chase et al., 2006). However, glycemia merely reflects three factors:

- Nutritional inputs (carbohydrate content in specific)

- Insulin (endogenous and exogenous)

- Insulin sensitivity $\left(S_{I}\right.$ hereafter $)$

A typical TGC control protocol controls only insulin dosing, excepting SPRINT which controls both insulin and nutrition. Many studies leave nutritional inputs to unit specific standards and/or don't report them. However, the glycemic response to be controlled is the response to both inputs.

Thus, insulin sensitivity is the primary factor. It determines the resulting glucose level for any given inputs, and thus how much insulin is required to achieve tight control, at least to the dose where insulin effect saturates (Natali et al., 2000). More specifically, in this model it accounts for the net affect of any suppression or increase in endogenous insulin and glucose production, and the rate of peripheral glucose uptake. Finally, the cytokines and hormones that drive these affects that result in hyperglycemia are physiologically linked to lowered insulin sensitivity and vary continuously over time as patient condition evolves. Hence, this overall effective insulin sensitivity is dynamic and time-varying.

\subsection{Insulin Sensitivity in the Critically Ill}

A clinically validated model is used to identify patientspecific, time-varying (hourly) insulin sensitivity every hour:

$$
\begin{array}{r}
\dot{G}=-p_{G} \cdot G-S_{I} \cdot G \cdot \frac{Q}{1+\alpha_{G} Q} \\
+\frac{P(t)+\left(P_{E N D} * m_{\text {body }}\right)-\left(C N S * m_{\text {brain }}\right)}{\left(V_{G, \text { frac }}(t) * m_{\text {body }}\right)} \\
\dot{Q}=-k Q+k I \\
\dot{I}=-\frac{n I}{1+\alpha_{I} I}+\frac{u_{e x}(t)}{\left(V_{I, \text { frac }} * m_{\text {body }}\right)}+e^{-\left(k_{I} \frac{u_{e x}(t)}{V_{i}}\right)} I_{B}
\end{array}
$$

Where $G(t)$ [mmol/L] is plasma glucose $I(t)[\mathrm{mmol} / \mathrm{L}]$ is plasma insulin, $u_{e x}(t)[\mathrm{mU} / \mathrm{min}]$ is exogenous insulin input, basal endogenous insulin secretion is $I_{B}[\mathrm{mU} / \mathrm{L} / \mathrm{min}]$, with $k_{I}$ representing suppression of basal insulin secretion by exogenous insulin. Interstitial insulin is $Q(t)$ [mU/L], with $k$ [1/min] accounting for losses and transport. Body weight and brain weight are denoted by $m_{\text {body }}[\mathrm{kg}]$ and $m_{\text {brain }}[\mathrm{kg}]$. Patient endogenous glucose clearance and insulin sensitivity are $p_{G}$ [1/min] and $S_{I}[\mathrm{~L} /(\mathrm{mU} . \mathrm{min})]$. The parameter $V_{I, \text { frac }}[\mathrm{L} / \mathrm{kg}]$ is the insulin distribution volume per $\mathrm{kg}$ body weight and $n$ $[1 / \mathrm{min}]$ is the transport rate of insulin from plasma. Total plasma glucose input is $P(t) \quad[\mathrm{mmol} / \mathrm{min}]$, endogenous glucose production is $P_{E N D}[\mathrm{mmol} / \mathrm{kg} / \mathrm{min}]$ and $V_{G \text {,frac }}[\mathrm{L} / \mathrm{kg}]$ represents the glucose distribution volume per $\mathrm{kg}$ body weight. CNS $[\mathrm{mmol} / \mathrm{kg} / \mathrm{min}]$ captures non-insulin mediated glucose uptake by the central nervous system. MichaelisMenten functions model saturation, with $\alpha_{I}[\mathrm{~L} / \mathrm{mU}]$ for the saturation of plasma insulin disappearance, and $\alpha_{G}[\mathrm{~L} / \mathrm{mU}]$ for insulin-dependent glucose clearance saturation.

These parameters and their clinically validated values are well documented in the literature (Lin et al., 2008). Additionally, they have been used in several clinical TGC studies including the development of SPRINT.

For this study, two cohorts are analysed:

- $\quad$ Adult ICU (ICU): $\mathrm{N}=384$ patients from SPRINT with over 40k hours of data (Chase et al., 2008b)

- Neonatal ICU (NICU): N = 25 patients and over 3500 hours of data (LeCompte et al., 2008)

For each cohort, insulin sensitivity, $S_{I}$, is identified hourly from the clinical data. In each cohort, the hour to hour variation in $S_{I}(t)$ for each patient is used to generate a stochastic model giving the probability distribution for hourly variation in $S_{I}$ from any current value of $S_{I}$ (Lin et al., 2008).

Fig. 1 shows the $S_{I}$ distribution for each cohort. It is clear that the NICU cohort has a far wider and flatter distribution of values. Theses results indicate a lesser level of whole body insulin resistance compared to adults. They also show a greater inter-patient variability in this parameter. For context, Fig. 1 also shows the typical values found in studies on type 2 diabetic individuals (Lotz, 2007).

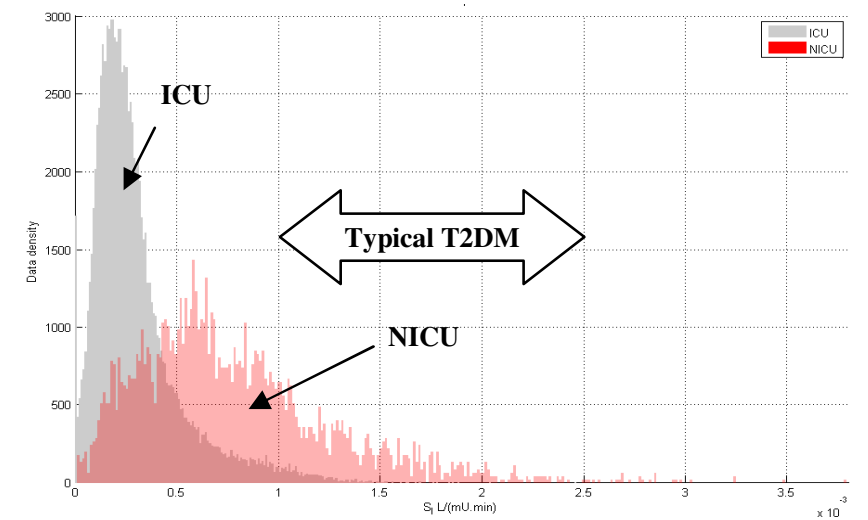

Fig. 1: ICU and NICU distributions of $S_{I}$. 
In detail, the ICU cohort, the median $S_{I-I C U}=0.22 \times 10^{-3}$ (IQR: [0.14-0.33] $\times 10^{-3}$; 90\%CI: [0.06-0.78] $\left.\times 10^{-3}\right)$ L/(mU.min). For the NICU cohort, median $S_{I-N I C U}=0.67 \times 10^{-3}$ (IQR: [0.43$\left.0.95] \times 10^{-3} ; 90 \% \mathrm{CI}: \quad[0.17-1.47] \times 10^{-3}\right) \quad$ L/(mU.min). For context, the range in healthy T2DM, $S_{\text {I-T2DM }}=[1,2.5] \times 10^{-3}$ L/(mU.min) (Lotz, 2007).

Fig. 2 shows the ICU and NICU stochastic models, capturing hourly variation from $S_{I, n}$ to $S_{I, n+l}$. The lines indicate the median, IQR and $90 \%$ CI for $S_{I, n+1}$ in the next hour on a vertical line from the current hour value, $S_{I, n}$ value on the Xaxis. Most variation is in a narrow band that grows wider with a downward skew, as $S_{I, n}$ rises (becoming more ill). Note the NICU axes are $\sim 2 \mathrm{x}$ larger.

Fig. 2 shows generally smaller variations at similar $S_{I, n}$ for the NICU case. Fig. 3 shows this variation in percent (from median) for each cohort in cumulative distribution functions. Median values for the 1-hour changes $\Delta S_{I-I C U}$ and $\Delta S_{I-N I C U}$ are zero $(\mathrm{p}<0.01)$. However, their IQR ranges are different $\left(\mathrm{IQR}: \Delta S_{I-N I C U}=[-7.5,+9.8] ; \Delta S_{I-I C U}=[-11.3,+15.7]\right) \%$ ( $\mathrm{p}=0.02$ ) with the IQR range of $\Delta S_{I-N I C U}$ is $40 \%$ smaller than the IQR range for $\Delta S_{I-I C U}$. The same results hold true for variations over 2, 3 and 4 hours, with the range of IQR for $\Delta S_{I}$ increasing over time to up to $60 \%$ (of median). Hence, adult ICU patients have greater intra-patient variation in $S_{I}$.
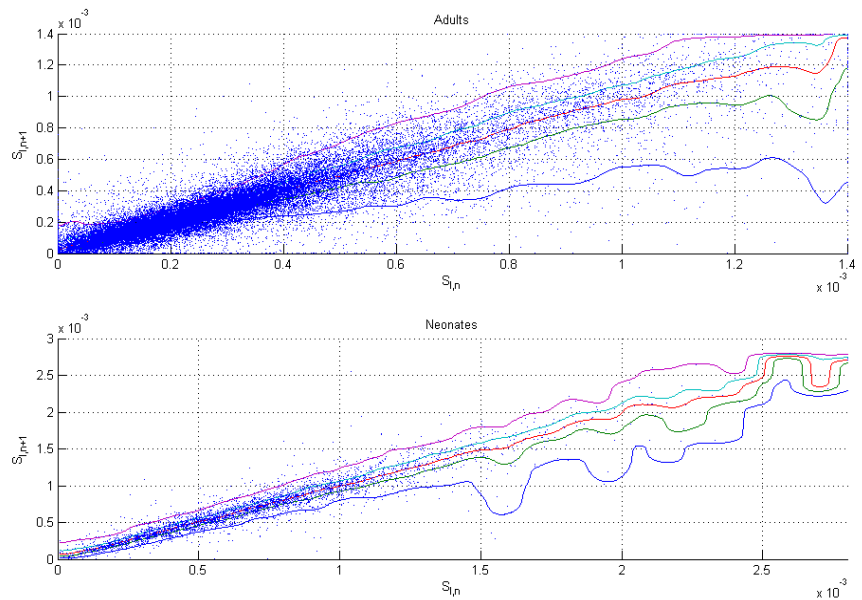

Fig. 2: ICU (top) and NICU (bottom) stochastic models showing hour to hour variation in $S_{I}$. Note axes are different.

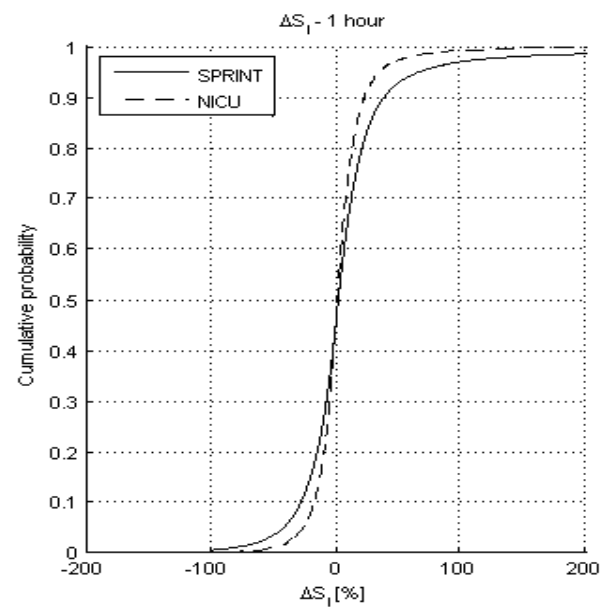

Fig. 3: 1-hourly variations (\%), $\Delta S_{I}$, for ICU and NICU.
Summary: Adult ICU patients have significantly more intrapatient variation in $S_{I}$ compared to NICU patients, and are thus far more dynamic in their evolution, which might be expected clinically. It is also clear ICU patients have far less inter-patient variability than NICU patients. Thus, each cohort has a significant form of variability to be managed.

\subsection{Summary and Implications for TGC Protocol Design}

This analysis of $S_{I}$ in two distinctly different critical care cohorts has significant implications for TGC protocols:

- $\quad$ To be patient specific a TGC protocol must directly (e.g. model-based) or indirectly account for both intra- and inter- patient variability.

- Measurement frequency must be 1-3 hourly and is likely to vary with patient condition and stability.

Currently, only a very few protocols either directly or indirectly adapt their intervention based on patient insulin sensitivity (Chase et al., 2008b, Wong et al., 2006, Plank et al., 2006, Braithwaite et al., 2006, Goldberg et al., 2004). Most are model-based. Some others, such as Clarian and Glucommander adjust based on insulin sensitivity, but are blinded to carbohydrate intake, so they only get a relative measure that is not full accurate. In further contrast, some clinical protocols account for increasing resistance (falling $S_{I}$ ) by increasing insulin dose, but do not formally adjust the other way, despite evidence of such changes in Fig 2 and other clinical studies. All of these issues are aggravated by often extended measurement periods out to 4-hourly, where variability can be quite wide, as evident from Figures 2-3.

Measurement frequency and clinical burden are major issues (Chase et al., 2008a). The IQR range for variations in $S_{I}$ at 3 and 4 hours approaches $60 \%$ ( $\pm \sim 30 \%)$, leading to significant variations in glycemic response for a given intervention. Given the prevalence of continuous infusions held over such longer intervals, even relatively modest variation (10-20\% over 3-4 hours) would result in significant changes in glucose from the intended outcome. Hence, as measurement periods rise so does both glycemic variability and hypoglycemia (Lonergan et al., 2006, Chase et al., 2006).

Given that some patients are more variable than others, failure to directly identify and account for patient variability means that some patients will receive, all else equal, more variable TGC. Thus, such clinical protocols are likely to fail in returning a mortality result, despite showing a good overall glycemic response for the cohort, as is seen comparing results across Table 1. Hence, fixed protocols that provide dosing based on more fixed parameters or protocols, rather than patient-specific responses are likely to fail.

For example, the NICU based NIRTURE trial (Beardsall et al., 2008) provided dosing on a fixed $\mathrm{mU} / \mathrm{g}$ body weight. They adjusted them on a fixed sliding scale to account for increasing insulin resistance but had little mechanism for lowering insulin dosage before hypoglycemia. As a result, it could not adapt to the wide range inter-patient variability in insulin sensitivity in neonates seen in Fig. 1, or to the modest intra-patient variability seen in Fig. 2. Long measurement 
periods out to 4-6 hours, with constant infusions in between exacerbated the problems with the resulting control. The end result was high glycemic variability with excessive hypoglycemia that stopped the trial early.

Similarly, the Leuven protocol was more successful with a less acutely ill and thus potentially less variable cardiovascular surgery cohort in 2001 than for the more critically ill medical ICU cohort in 2006. This protocol also had similar elements of long measurement periods based on patient glucose levels, rather than any indication of stability. In contrast, SPRINT increases measurement intervals only after several measurements in a tight band at pre-set levels of interventions that indicate high insulin sensitivity. The difference is in adjusting based on patient stability in both glycemic response and insulin sensitivity, rather than on a particular glucose level.

Lonergan et al (2006) demonstrated these results in a protocol comparison using a validated virtual trial approach. The results indicated that any system that did not adapt intervention and/or measurement period based on patientspecific metrics, as do model-based and similar systems (e.g. SPRINT), would be unable to provide the same level of glycemic control for all patients. Thus, the primary implication is simply that for TGC to provide equal control to all patients, the control protocol must also be patient-specific.

Finally, most protocols that have not yielded full success are typically blinded to the nutritional intake. Thus, they cannot be patient-specific. In particular, it is the interaction between insulin sensitivity (resistance ${ }^{-1}$ ), insulin and nutrition that determines glycemic outcome. Not knowing one of these variables means patient-specific control cannot be delivered.

Summary: A TGC cohort result may have acceptable median and variability, as seen in Table 1, but the clinical outcome will be highly dependent on how each patient is treated. Failure to account for inter- and intra- patient variability will result in poor TGC for the more dynamic patients (intrapatient variability) or those for whom dosing is inappropriate due to inter-patient variability. Managing variability means that any protocol must be able to adapt and provide patient specific interventions that evolve with patient condition. Finally, measurement frequencies must be short enough to minimise potential variation between interventions. At 1-3 hours for maximum sampling periods, protocols must also be designed to minimise clinical burden. Failing these issues, glycemic markers may not be eliminated as a marker for mortality, thus rendering any further clinical comparisons difficult or not valid.

\section{CONCLUSIONS}

The field of critical care has seen a great deal of debate over TGC therapy and its efficacy. This article has attempted to show that much of the difficulty may be due to incorrect targets in creating TGC protocols or applying TGC therapy, by using a cohort based measure of glycemic control to see changes in mortality, which is an individual outcome. Secondly, it shows that the difficulty in translating cohortbased control to individual outcomes is likely the result of patient variability, as seen by variations in insulin sensitivity.
Finally, both parts of the paper outlined distinct metrics and/or goals, based on this analysis and prior successful results, to provide potential directions and goals for designing and implementing the next generation of TGC protocols.

\section{REFERENCES}

Ahrens, C. L., Barletta, J. F., Kanji, S., Tyburski, J. G., Wilson, R. F., Janisse, J. J. \& Devlin, J. W. (2005) Effect of low-calorie parenteral nutrition on the incidence and severity of hyperglycemia in surgical patients: a randomized, controlled trial. Crit Care Med, 33, 2507-12.

Beardsall, K., Vanhaesebrouck, S., Ogilvy-Stuart, A. L., Vanhole, C., Palmer, C. R., Van Weissenbruch, M., Midgley, P., Thompson, M., Thio, M., Cornette, L., Ossuetta, I., Iglesias, I., Theyskens, C., De Jong, M., Ahluwalia, J. S., De Zegher, F. \& Dunger, D. B. (2008) Early insulin therapy in very-low-birth-weight infants. N Engl J Med, 359, 1873-84.

Bistrian, B. R. (2001) Hyperglycemia and Infection: Which is the Chicken and Which is the Egg? JPEN J Parenter Enteral Nutr, 25, 180-181.

Braithwaite, S. S., Edkins, R., Macgregor, K. L., Sredzienski, E. S., Houston, M., Zarzaur, B., Rich, P. B., Benedetto, B. \& Rutherford, E. J. (2006) Performance of a dosedefining insulin infusion protocol among trauma service intensive care unit admissions. Diabetes Technol Ther, 8, 476-88.

Brunkhorst, F. M., Engel, C., Bloos, F., Meier-Hellmann, A., Ragaller, M., Weiler, N., Moerer, O., Gruendling, M., Oppert, M., Grond, S., Olthoff, D., Jaschinski, U., John, S., Rossaint, R., Welte, T., Schaefer, M., Kern, P., Kuhnt, E., Kiehntopf, M., Hartog, C., Natanson, C., Loeffler, M. \& Reinhart, K. (2008) Intensive insulin therapy and pentastarch resuscitation in severe sepsis. $N$ Engl J Med, 358, 125-39.

Capes, S. E., Hunt, D., Malmberg, K. \& Gerstein, H. C. (2000) Stress hyperglycaemia and increased risk of death after myocardial infarction in patients with and without diabetes: a systematic overview. Lancet, 355, 773-778.

Chase, J., Andreassen, S., Jensen, K. \& Shaw, G. (2008a) The Impact of Human Factors on Clinical Protocol Performance - A proposed assessment framework and case examples. Journal of Diabetes Science and Technology (JoDST), 2, 409-416.

Chase, J., Shaw, G. M., Wong, X. W., Lotz, T., Lin, J. \& Hann, C. E. (2006) Model-based Glycaemic Control in Critical Care - A review of the state of the possible. Biomedical Signal Processing and Control, 1, 3-21.

Chase, J. G., Shaw, G., Le Compte, A., Lonergan, T., Willacy, M., Wong, X. W., Lin, J., Lotz, T., Lee, D. \& Hann, C. (2008b) Implementation and evaluation of the SPRINT protocol for tight glycaemic control in critically ill patients: a clinical practice change. Crit Care, 12, R49.

Das, U. N. (2003) Insulin in sepsis and septic shock. J Assoc Physicians India, 51, 695-700.

De La Rosa, C., Donado, J. H., Restrepo, A. H., Quintero, A. M., Gonzalez, L. G., Saldarriaga, N. E., Bedoya, M., 
Toro, J. M., Velasquez, J. B., Valencia, J. C., Arango, C. M., Aleman, P. H., Vasquez, E. M., Chavarriaga, J. C., Yepes, A., Pulido, W. \& Cadavid, C. A. (2008) Strict glycaemic control in patients hospitalised in a mixed medical and surgical intensive care unit: a randomised clinical trial. Crit Care, 12, R120.

Devos, P. \& Preiser, J. C. (2007) Current controversies around tight glucose control in critically ill patients. Curr Opin Clin Nutr Metab Care, 10, 206-9.

Egi, M., Bellomo, R., Stachowski, E., French, C. J. \& Hart, G. (2006) Variability of blood glucose concentration and short-term mortality in critically ill patients. Anesthesiology, 105, 244-52.

Fernandez-Real, J. M., Broch, M., Richart, C., Vendrell, J., Lopez-Bermejo, A. \& Ricart, W. (2003) CD14 monocyte receptor, involved in the inflammatory cascade, and insulin sensitivity. The Journal of clinical endocrinology and metabolism, 88, 1780-1784.

Finfer, S. \& Delaney, A. (2008) Tight glycemic control in critically ill adults. Jama, 300, 963-5.

Goldberg, P. A., Sakharova, O. V., Barrett, P. W., Falko, L. N., Roussel, M. G., Bak, L., Blake-Holmes, D., Marieb, N. J. \& Inzucchi, S. E. (2004) Improving glycemic control in the cardiothoracic intensive care unit: clinical experience in two hospital settings. J Cardiothorac Vasc Anesth, 18, 690-7.

Krinsley, J. S. (2003) Association between hyperglycemia and increased hospital mortality in a heterogeneous population of critically ill patients. Mayo Clin Proc, 78, 1471-1478.

Krinsley, J. S. (2008) Glycemic variability: a strong independent predictor of mortality in critically ill patients. Crit Care Med, 36, 3008-13.

Krishnan, J. A., Parce, P. B., Martinez, A., Diette, G. B. \& Brower, R. G. (2003) Caloric intake in medical ICU patients: consistency of care with guidelines and relationship to clinical outcomes. Chest, 124, 297-305.

Lecompte, A., Chase, J., Shaw, G., Lynn, A. \& Russell, G. (2008) Blood glucose modelling and control for preterm infants. 2008 Engineering \& Physical Sciences in Medicine and Australian Biomedical Engineering Conference (EPSM ABEC 2008). Christchurch, New Zealand, ABEC.

Lin, J., Lee, D., Chase, J. G., Shaw, G. M., Le Compte, A., Lotz, T., Wong, J., Lonergan, T. \& Hann, C. E. (2008) Stochastic modelling of insulin sensitivity and adaptive glycemic control for critical care. Comput Methods Programs Biomed, 89, 141-52.

Lonergan, T., Lecompte, A., Willacy, M., Chase, J. G., Shaw, G. M., Wong, X. W., Lotz, T., Lin, J. \& Hann, C. E. (2006) A Simple Insulin-Nutrition Protocol for Tight Glycemic Control in Critical Illness: Development and Protocol Comparison. Diabetes Technol Ther, 8, 191206.

Lotz, T. (2007) High Resolution Clinical Model-Based Assessment of Insulin Sensitivity. Mechanical Engineering. Christchurch, University of Canterbury.

Marik, P. E. \& Raghavan, M. (2004) Stress-hyperglycemia, insulin and immunomodulation in sepsis. Intensive Care Medicine, 30, 748-756.
Mccowen, K. C., Malhotra, A. \& Bistrian, B. R. (2001) Stress-induced hyperglycemia. Crit Care Clin, 17, 107124.

Meijering, S., Corstjens, A. M., Tulleken, J. E., Meertens, J. H., Zijlstra, J. G. \& Ligtenberg, J. J. (2006) Towards a feasible algorithm for tight glycaemic control in critically ill patients: a systematic review of the literature. Crit Care, 10, R19.

Mesotten, D. (2008) Tight glycaemic control in the intensive care unit: pitfalls in the testing of the concept. Crit Care, 12, 187.

Natali, A., Gastaldelli, A., Camastra, S., Sironi, A. M., Toschi, E., Masoni, A., Ferrannini, E. \& Mari, A. (2000) Dose-response characteristics of insulin action on glucose metabolism: a non-steady-state approach. Am J Physiol Endocrinol Metab, 278, E794-801.

Plank, J., Blaha, J., Cordingley, J., Wilinska, M. E., Chassin, L. J., Morgan, C., Squire, S., Haluzik, M., Kremen, J., Svacina, S., Toller, W., Plasnik, A., Ellmerer, M., Hovorka, R. \& Pieber, T. R. (2006) Multicentric, randomized, controlled trial to evaluate blood glucose control by the model predictive control algorithm versus routine glucose management protocols in intensive care unit patients. Diabetes Care, 29, 271-6.

Treggiari, M. M., Karir, V., Yanez, N. D., Weiss, N. S., Daniel, S. \& Deem, S. A. (2008) Intensive insulin therapy and mortality in critically ill patients. Crit Care, 12, R29.

Umpierrez, G. E., Isaacs, S. D., Bazargan, N., You, X., Thaler, L. M. \& Kitabchi, A. E. (2002) Hyperglycemia: an independent marker of in-hospital mortality in patients with undiagnosed diabetes. J Clin Endocrinol Metab, 87, 978-982.

Van Den Berghe, G., Wouters, P., Weekers, F., Verwaest, C., Bruyninckx, F., Schetz, M., Vlasselaers, D., Ferdinande, P., Lauwers, P. \& Bouillon, R. (2001) Intensive insulin therapy in the critically ill patients. $N$ Engl J Med, 345, 1359-1367.

Van Den Berghe, G., Wouters, P. J., Bouillon, R., Weekers, F., Verwaest, C., Schetz, M., Vlasselaers, D., Ferdinande, P. \& Lauwers, P. (2003) Outcome benefit of intensive insulin therapy in the critically ill: Insulin dose versus glycemic control. Crit Care Med, 31, 359366.

Weekers, F., Giulietti, A. P., Michalaki, M., Coopmans, W., Van Herck, E., Mathieu, C. \& Van Den Berghe, G. (2003) Metabolic, endocrine, and immune effects of stress hyperglycemia in a rabbit model of prolonged critical illness. Endocrinology, 144, 5329-38.

Wilson, M., Weinreb, J. \& Hoo, G. W. (2007) Intensive insulin therapy in critical care: a review of 12 protocols. Diabetes Care, 30, 1005-11.

Wong, X. W., Singh-Levett, I., Hollingsworth, L. J., Shaw, G. M., Hann, C. E., Lotz, T., Lin, J., Wong, O. S. \& Chase, J. G. (2006) A novel, model-based insulin and nutrition delivery controller for glycemic regulation in critically ill patients. Diabetes Technol Ther, 8, 174-90. 